\title{
Second-harmonic optical spectroscopy on split-ring-resonator arrays
}

\author{
Fabian B. P. Niesler, ${ }^{1, *}$ Nils Feth, ${ }^{2}$ Stefan Linden, ${ }^{1,2,3}$ and Martin Wegener ${ }^{1,2,3}$ \\ ${ }^{1}$ Institut für Angewandte Physik, Karlsruhe Institute of Technology (KIT), D-76128 Karlsruhe, Germany \\ ${ }^{2}$ Institut für Nanotechnologie, Karlsruhe Institute of Technology (KIT), \\ Hermann-von-Helmholtz-Platz 1, D-76344 Eggenstein-Leopoldshafen, Germany \\ ${ }^{3}$ DFG-Center for Functional Nanostructures (CFN), Karlsruhe Institute of Technology (KIT), D-76128 Karlsruhe, Germany \\ ${ }^{*}$ Corresponding author: fabian.niesler@kit.edu
}

Received December 22, 2010; revised March 4, 2011; accepted March 24, 2011; posted March 30, 2011 (Doc. ID 140121); published April 20, 2011

\begin{abstract}
Previous second-harmonic-generation experiments on metallic split-ring-resonator arrays have been performed at fixed fundamental laser center frequency. Here, we perform nonlinear optical spectroscopy on a first set of samples, revealing pronounced resonances. Furthermore, to clarify the role of higher-order split-ring resonances, we perform additional experiments on a second set of samples in which the fundamental split-ring-resonator resonance frequencies are lithographically tuned, whereas the higher-order resonances are fixed. We find that the higher-order resonances merely reabsorb the second-harmonic generation, revealing the fundamental split-ring resonance as the nonlinear source. (c) 2011 Optical Society of America

OCIS codes: $190.0190,160.3918$.
\end{abstract}

Split-ring resonators are the basis of high-frequency magnetism in negative-index metamaterials [1,2], of optical activity and huge circular dichroism in chiral metamaterials $[3, \underline{4}]$, and potentially of large optical nonlinearities in nonlinear metamaterials [5-7]. Previous nonlinear optical experiments [5-7] have explored the intrinsic metal nonlinearity as well as nonlinearities of adjacent materials, such as GaAs, enhanced by local-field effects [8]. Regarding the former, the precise source of the optical nonlinearity is still the subject of debate. Specifically, volume contributions as well as surface contributions have been discussed theoretically [9]. In order to eventually resolve this issue, it is highly desirable to provide further experimental data. Specifically, previous experiments on split-ring resonators (SRRs) [5-7] and on related structures $[10,11]$ were all performed at fixed fundamental laser frequency, leaving plenty of room for speculation.

In this Letter, we perform nonlinear optical spectroscopy of second-harmonic generation (SHG) on split-ring-resonator arrays. These spectroscopic data immediately clarify the role of higher-order resonances and will likely provide a much more sensitive future testing ground for microscopic theories of the underlying mechanisms.

The gold SRR samples on glass substrate used in our experiments are fabricated by means of standard electron-beam lithography (for details see, e.g., Refs. [5,12]). The footprint size of the SRR arrays is $200 \mu \mathrm{m} \times 2 \overline{00 \mu \mathrm{m}}$. These samples are excited under normal incidence and with horizontally polarized incident light, for which the fundamental resonance of the SRR is allowed. As shown previously [5] and as expected from symmetry, the SHG signal emerges with vertical linear polarization. As the excitation source, we employ an optical parametric amplifier (OPA, Spectra Physics OPA $800 \mathrm{CF}$ ) that is tunable from 1.2 to $1.56 \mu \mathrm{m}$ wavelength and that is pumped by a regeneratively amplified Ti:sapphire femtosecond laser (Spectra Physics Hurricane). The OPA delivers pulses with a duration of about $150 \mathrm{fs}$ at a repetition rate of $1 \mathrm{kHz}$.

The output of the OPA is attenuated and focused onto the SRR samples from the air side under normal incidence to a Gaussian spot diameter on the order of $60 \mu \mathrm{m}$. The SHG signal emerging in the forward direction through the glass substrate is sent into a grating spectrometer that is connected to a sensitive liquid-nitrogencooled CCD silicon-based camera. The plotted SHG

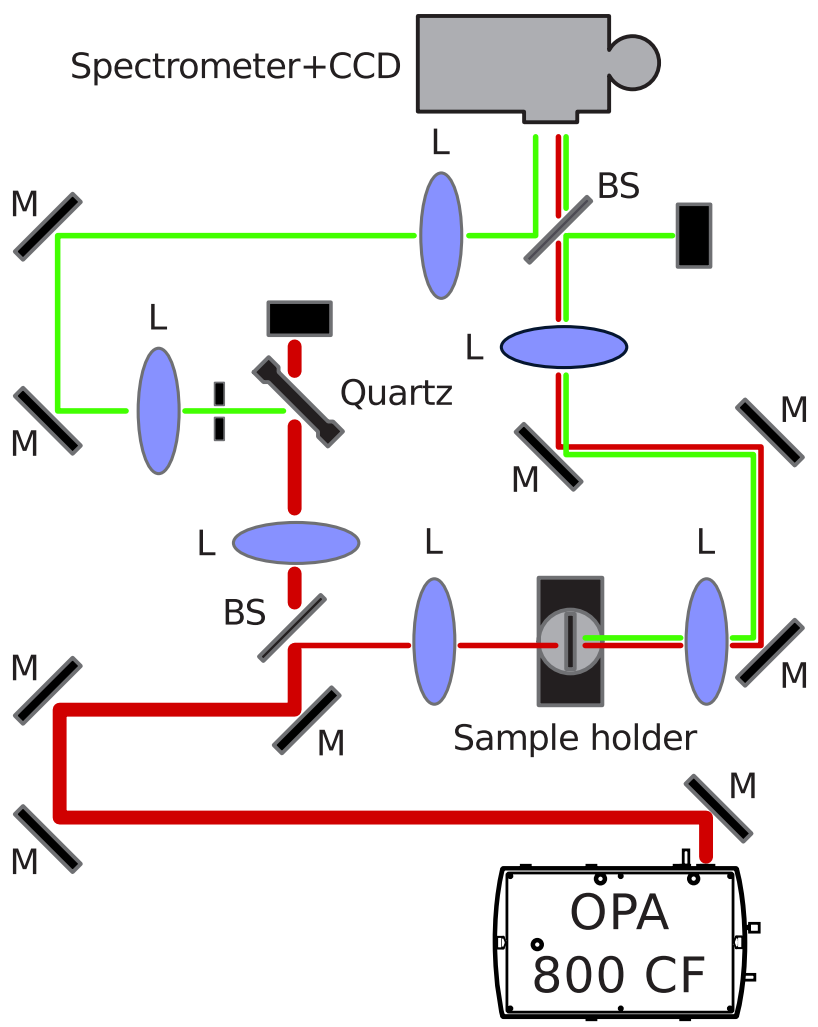

Fig. 1. (Color online) Scheme of the experimental setup for spectrally resolved nonlinear optical second-harmonic generation from split-ring-resonator arrays (M: mirror, L: lens, BS: beam splitter). 
signals are obtained by integrating over the resulting spectral peak centered at around half the fundamental laser wavelength. When tuning the OPA, its average power, its pulse duration, its beam divergence, and its spot diameter can typically change quite considerably. Thus, the focus conditions on the SRR sample change substantially. To still acquire reproducible and meaningful SHG spectra, we normalize the SHG signal from the SRR array to the SHG obtained from the surface of a quartz crystal plate excited under an angle located in a second arm of our experimental setup. In doing so, we assume that the quartz surface SHG exhibits no significant spectral dependence, which we believe is reasonable considering its bandgap in the UV region at around $8.4 \mathrm{eV}$ [13] photon energy (equivalent to $148 \mathrm{~nm}$ wavelength). In this fashion, we obtain reproducible experimental results. The normalization is the same for all samples and conditions in this Letter, such that the SHG
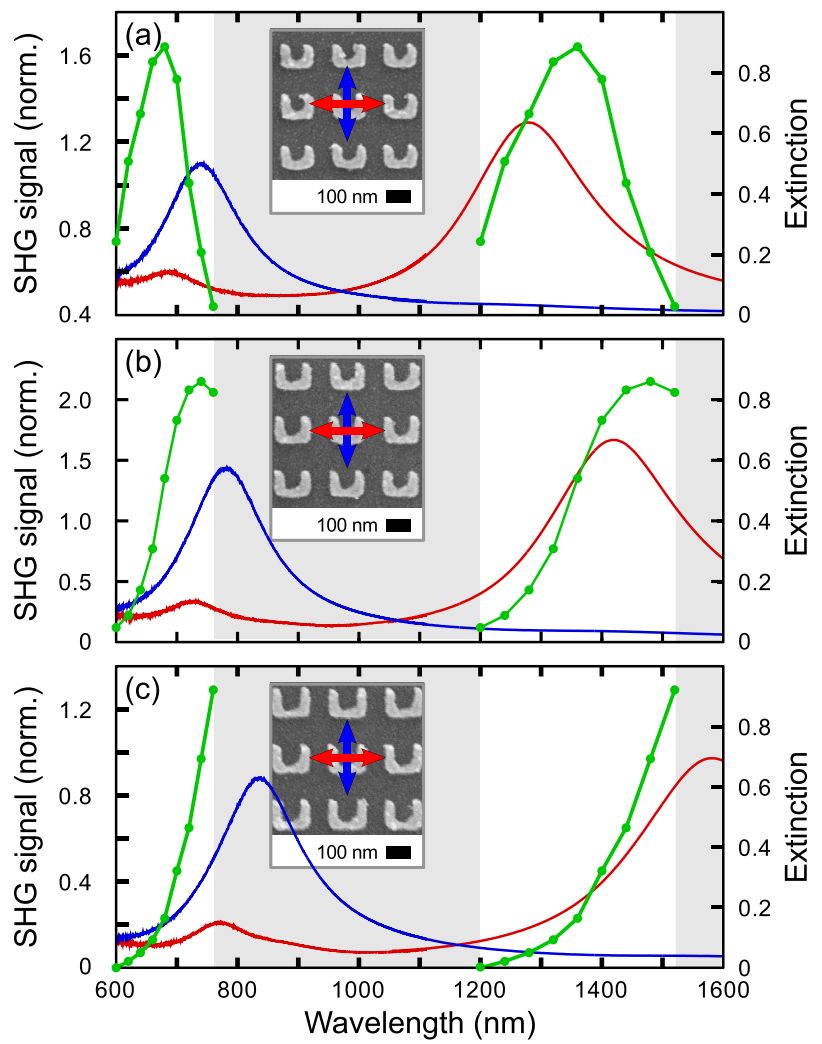

Fig. 2. (Color online) Second-harmonic-generation spectra (dots connected by green curves to guide the eye) obtained from three different samples (a)-(c) with different SRR sizes. The incident fundamental laser is linearly polarized along the horizontal direction as defined with respect to the samples by the red double arrows. The SHG emerges with vertical linear polarization as defined by the blue double arrows. Each SHG data point is intentionally plotted twice in the same spectrum: as a function of the fundamental laser wavelength and as a function of half that wavelength (i.e., the SHG wavelength). Spectral regions that are not accessible by the OPA tuning range are indicated in gray. To define the samples, the insets in (a)-(c) show electron micrographs for each sample. To allow for direct comparison with the SHG data, normal incidence linear extinction spectra (negative logarithm of the measured transmittance) for horizontal (red) and vertical (blue) incident linear polarization are also shown for each case (a)-(c). signal strengths in Figs. 2 and 3 can be compared directly. At the laser powers used (below $500 \mu \mathrm{W}$ average power, equivalent to $0.5 \mu \mathrm{J}$ pulse energy, or $3.3 \mathrm{MW}$ peak power, or about $100 \mathrm{GW} \mathrm{cm}^{-2}$ peak intensity on the sample), the SHG signal closely scales with the square of the incident power (not depicted). Furthermore, we find no detectable deterioration of the gold SRR samples during and/ or after our experiments that often take several days. The experimental setup is schematically shown in Fig. 1.

Split-ring resonators show several distinct resonances as, e.g., discussed in Refs. [ $[\underline{12}]$. In essence, the fundamental mode has an oscillating and circulating electric current with nodes only at the two ends of the wire, whereas the higher-order resonances are standing waves of the current with additional current nodes. Typically, the next-higher-order resonances are very roughly at twice the frequency of the fundamental (magnetic) resonance frequency. Thus, it is not a priori clear at all which of these resonances governs the SHG. Previous
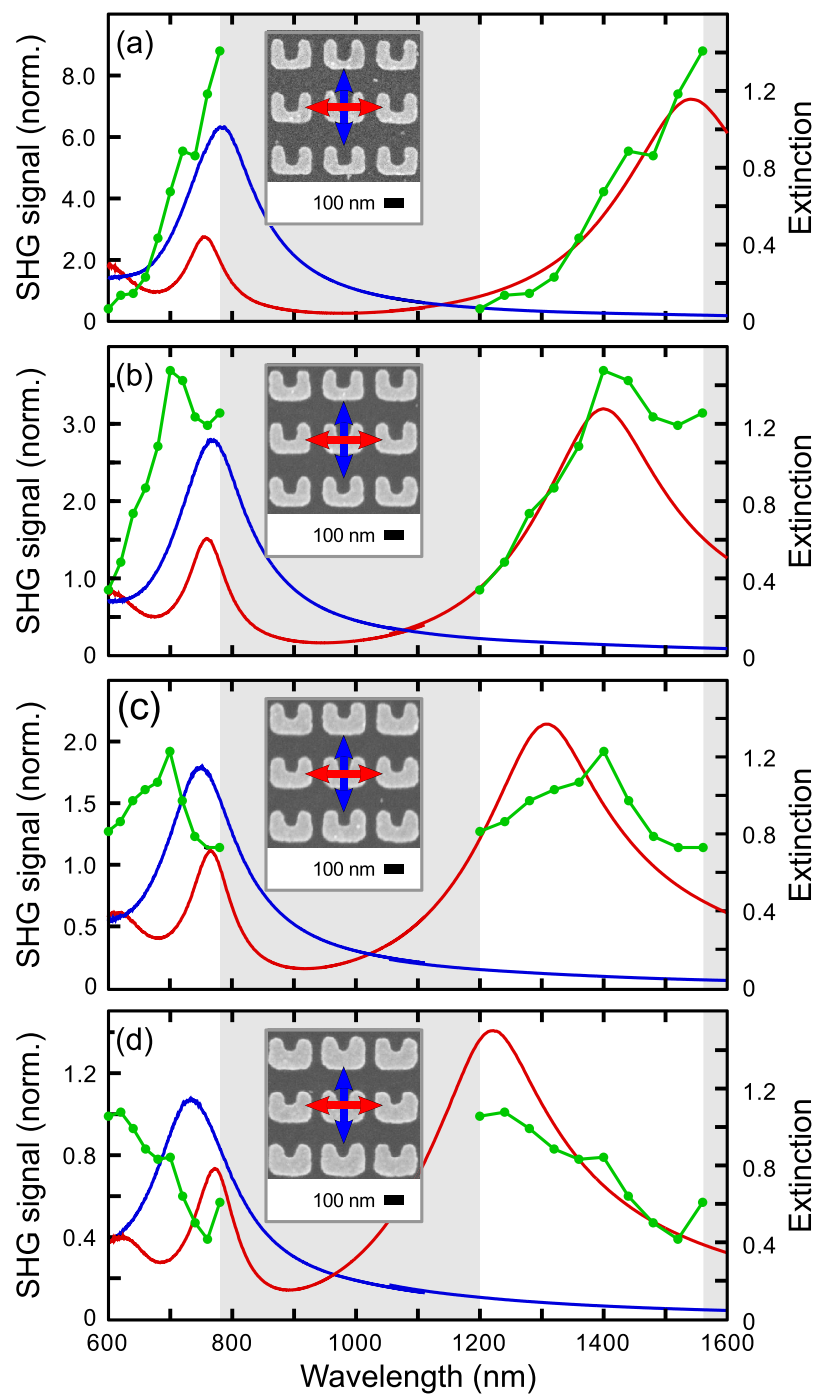

Fig. 3. (Color online) Experimental results represented as in Fig. 2, but for a second set of SRR samples for which the shape (rather than just the size) is lithographically tuned. The shape tuning is performed such that the fundamental SRR resonances shift, whereas the higher-order resonances are approximately fixed. 
experiments could not really rule out the possibility of a double-resonance situation either [5-7]. Thus, we plot all of our measured SHG signal data points in two different ways: as a function of the fundamental laser frequency and as a function of the SHG wavelength, i.e., each data point intentionally appears twice in the plots. Corresponding data for three SRR samples with different sizes are depicted in Fig. 2. The linear optical extinction spectra (negative logarithm of the measured normal incidence intensity transmittance) for horizontal and vertical incident polarization of light are shown for reference. Unity extinction corresponds to $10 \%$ transmittance. Clearly, the SHG signal reveals a maximum that shifts when changing the SRR size and, hence, the SRR resonance positions. The SHG maximum is slightly redshifted with respect to that of the linear extinction maximum.

As pointed out above, it is not quite clear from the data in Fig. 2 alone whether the SHG maximum correlates with the SRR fundamental resonance frequency, with the higher-order resonances, or with both. To further investigate this aspect, we have fabricated a second set of samples in which the shape (!) of the SRR is systematically changed in such a way that the fundamental SRR resonance is lithographically tuned, whereas the higher-order resonances are approximately fixed. Corresponding electron micrographs are depicted in Fig. 3. Obviously, the depth of the notch in the U-shaped SRR varies. The corresponding linear and nonlinear optical spectra are represented as in Fig. 2. Again, we find SHG resonances. However, the comparison with the linear extinction spectra in Fig. 3 shows that the SHG maximum is generally not at the same position as the higher-order resonances. For example, in Fig. 3(b) the linear extinction for vertical polarization (blue) peaks about $770 \mathrm{~nm}$ wavelength. At this wavelength, the vertically polarized SHG signal (green) exhibits a minimum. The same holds true in Fig. 3(c) and 3(d) with very little spectral shift. In contrast, the linear extinction maximum for vertical polarization (red) in Fig. 3 gradually shifts from $1540 \mathrm{~nm}$ in (a) to $1220 \mathrm{~nm}$ in (d). The SHG maximum versus fundamental laser wavelength shifts accordingly. We interpret this behavior as indicating that the fundamental SRR resonance acts as the nonlinear source and that the higherorder SRR resonances reabsorb the generated vertically polarized SHG.

In conclusion, we have performed nonlinear optical spectroscopy on arrays of gold split-ring resonators. The resulting spectra show a pronounced resonance maximum of second-harmonic generation that correlates with the fundamental resonance but not with the higherorder resonance. We hope that these spectroscopic data will help clarify the underlying nonlinear mechanism ("bulk" or "surface") by comparison with results from microscopic theory, which, however, are presently not yet available.

We thank Kurt Busch and his group for discussions. We acknowledge support by the Deutsche Forschungsgemeinschaft (DFG) and the State of Baden-Württemberg through the DFG-Center for Functional Nanostructures (CFN) within subproject A 1.5. The project PHOME acknowledges the financial support of the Future and Emerging Technologies program within the Seventh Framework Programme for Research of the European Commission, under FET-Open grant number 213390. The project METAMAT is supported by the Bundesministerium für Bildung und Forschung. The research of S. Linden is further supported through a HelmholtzHochschul-Nachwuchsgruppe (VH-NG-232). The Ph.D. education of F. B. P. Niesler is embedded in the Karlsruhe School of Optics and Photonics.

\section{References}

1. V. M. Shalaev, Nat. Photon. 1, 41 (2007).

2. C. M. Soukoulis, S. Linden, and M. Wegener, Science $\mathbf{3 1 5}$, 47 (2007).

3. A. V. Rogacheva, V. A. Fedotov, A. S. Schwanecke, and N. I. Zheludev, Phys. Rev. Lett. 97, 177401 (2006).

4. M. Decker, R. Zhao, C. M. Soukoulis, S. Linden, and M. Wegener, Opt. Lett. 35, 1593 (2010).

5. M. W. Klein, C. Enkrich, M. Wegener, and S. Linden, Science 313, 502 (2006)

6. M. W. Klein, N. Feth, S. Linden, and M. Wegener, Opt. Express 15, 5238 (2007).

7. N. Feth, S. Linden, M. W. Klein, M. Decker, F. B. P. Niesler, Y. Zeng, W. Hoyer, J. Liu, S. W. Koch, J. V. Moloney, and M. Wegener, Opt. Lett. 33, 1975 (2008).

8. F. B. P. Niesler, N. Feth, S. Linden, J. Niegemann, J. Gieseler, K. Busch, and M. Wegener, Opt. Lett. 34, 1997 (2009).

9. Y. Zeng and J. V. Moloney, Opt. Lett. 34, 2844 (2009).

10. S. Kujala, B. K. Canfield, M. Kauranen, Y. Svirko, and J. Turunen, Phys. Rev. Lett. 98, 167403 (2007).

11. B. K. Canfield, H. Husu, J. Laukkanen, B. Bai, M. Kuittinen, J. Turunen, and M. Kauranen, Nano Lett. 7, 1251 (2007).

12. S. Linden, C. Enkrich, M. Wegener, J. F. Zhou, T. Koschny, and C. M. Soukoulis, Science 306, 1351 (2004).

13. W. H. Strehlow and E. L. Cook, J. Phys. Chem. Ref. Data 2, 163 (1973). 